\title{
Подавление электронно-дырочного обменного взаимодействия в резервуаре неизлучающих экситонов
}

\author{
() А.В. Трифоонов, И.В. Игнатьев , К.В. Кавокин, А.В. Кавокин, \\ П.Ю. Шапочкин, Ю.П. Ефримов, С.А. Елисеев, В.А. Ловцюс
}

Санкт-Петербургский государственный университет, 198504 Санкт-Петербург, Россия

๑ E-mail: i.ignatiev@spbu.ru

Поступила в Редакцию 24 апреля 2019 г. В окончательной редакции 29 апреля 2019 г. Принята к публикации 29 апреля 2019 г.

\begin{abstract}
Выполнен теоретический анализ механизмов подавления электронно-дырочного обменного взаимодействия в неизлучающих экситонах с большим волновым вектором в высококачественных гетероструктурах с квантовыми ямами. Показано, что основным механизмом подавления является рассеяние экситонов с взаимным переворотом спинов носителей одного знака (либо двух электронов, либо двух дырок), входящих в экситоны. В результате этого спиновая поляризация электронов в неизлучающих экситонах может сохраняться длительное время. Анализ экспериментальных результатов показывает, что это время может быть больше одной наносекунды. Столь длительная и оптически контролируемая спиновая память в экситонном резервуаре может представлять интерес для будущих информационных технологий.
\end{abstract}

Ключевые слова: экситон, обменное взаимодействие, квантовая яма.

DOI: 10.21883/FTP.2019.09.48123.06

\section{1. Введение}

В работе [1] было продемонстрировано, что при оптическом возбуждении высококачественных гетероструктур с квантовыми ямами на основе прямозонных полупроводников типа GaAs возможно накопление резервуара неизлучающих экситонов с волновым вектором, превышающим волновой вектор света в материале квантовой ямы. Плотность экситонов в этом резервуаре может на несколько десятичных порядков превышать плотность излучающих экситонов с волновым вектором, находящимся в пределах светового конуса. Большая разница плотностей излучающих и неизлучающих экситонов связана с разницей их времен жизни. Излучательное время покоящихся экситонов является очень коротким, $\sim 10$ пс. В то же время неизлучающие экситоны в высококачественных гетероструктурах могут жить десятки наносекунд [1].

Хотя неизлучающие экситоны не взаимодействуют со светом, их оптическое зондирование возможно по тем эффектам, которые они оказывают на излучающие экситоны. В частности, рассеяние излучающих и неизлучающих экситонов приводит к уширению и энергетическому сдвигу экситонных резонансов в спектрах отражения. Более того, наши недавние исследования показали [2], что сдвиг экситонных состояний является поляризационно-чувствительным. При возбуждении образца циркулярно-поляризованным излучением сдвиг экситонных резонансов оказывается различным в ко- и кросс-циркулярных поляризациях детектирования. Это указывает на сохранение спиновой поляризации в резервуаре неизлучающих экситонов.
В работе [2] исследована динамика спиновой поляризации экситонного резервуара методом накачка-зондирование со спектральным и поляризационным разрешением в магнитном поле, приложенном поперек оси роста гетероструктуры (геометрия Фохта). Изучалась высококачественная гетероструктура с квантовой ямой $\mathrm{GaAs}$ шириной 14 нм, выращенная методом молекулярно-пучковой эпитаксии. Пример спектра отражения этой гетероструктуры в области оптических переходов на размерно-квантованные состояния тяжелых (Xhh) легких (Xlh) экситонов приведен на рис. 1. Наблюдаемые экситонные резонансы хорошо описываются в рамках стандартной модели отражения [3-6]. Это позволяет с высокой точностью определять спектральное положение и уширение экситонных резонансов. При возбуждении импульсами накачки экситонный резонанс уширяется и немного сдвигается, что позволяет изучать динамику сдвига и уширения с помощью регистрации спектров отражения пробных импульсов, задержанных во времени относительно импульсов накачки. Пример таких зависимостей для резонанса Xhh показан на рис. 2. Как видно из рисунков, в момент прихода импульса накачки наблюдается резкий красный сдвиг экситонного резонанса и его значительное уширение. Затем наблюдается относительно медленное уменьшение сдвига и избыточного уширения на временах порядка нескольких наносекунд. Эти закономерности объясняются рождением неизлучающих экситонов импульсом накачки и затем постепенным опустошением резервуара этих экситонов [2].

При приложении поперечного магнитного поля (геометрия Фохта) и возбуждении циркулярно поляризованными импульсами накачки в спектре отражения 


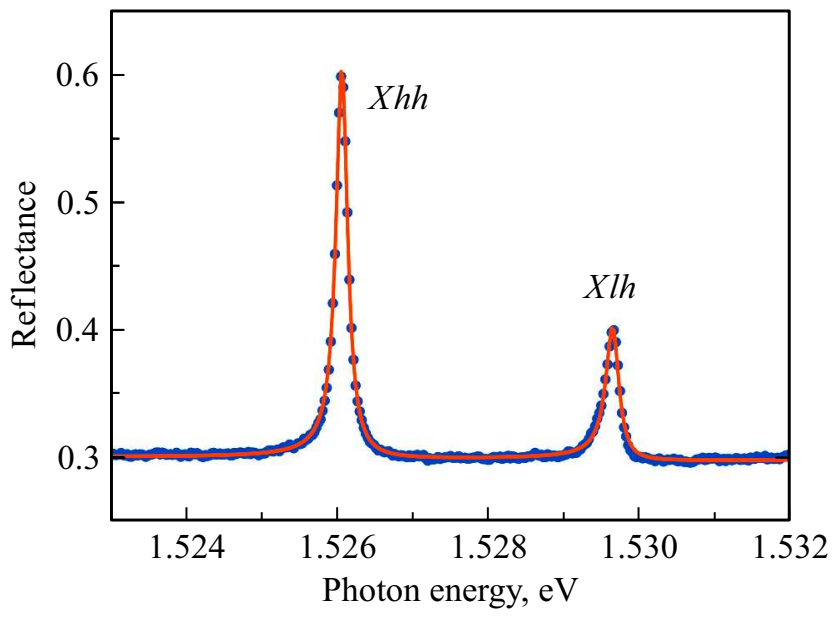

Рис. 1. Спектры отражения образца с квантовой ямой 14 нм (точки) и его подгонка в рамках стандартной модели (сплошная кривая). Хорошо видны резонансы отражения, соответствующие оптическим переходам с рождением тяжелого (Xhh) и легкого $(\mathrm{Xlh})$ экситонов. Температура образца $T=6 \mathrm{~K}$.
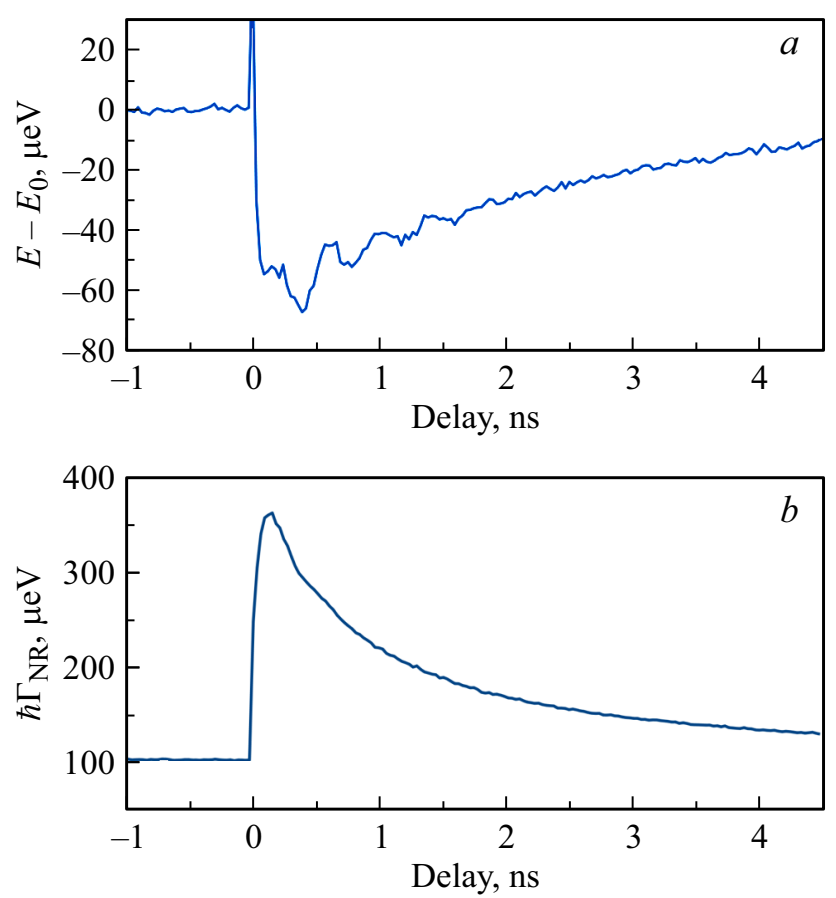

Рис. 2. Сдвиг энергии тяжелого экситона $(a)$ и уширение экситонного резонанса Xhh $(b)$ как функции задержки между импульсами накачки и зондирования. Магнитное поле $B_{x}=0.5$ Тл. Хорошо видны осцилляции энергетического положения экситона (панель $a$ ), которые отсутствуют в динамике уширения (панель $b$ ).

наблюдается осциллирующая во времени компонента сдвига экситонного резонанса (см. рис. 2, $a$ ). Фаза осцилляций сдвига противоположна при детектировании спектров в ко- и кросс-поляризациях по отношению к поляризации накачки. Пример разницы этих сдвигов показан на рис. 3. Экспериментально установлено, что частота осцилляций линейно зависит от приложенного магнитного поля, $\hbar \omega=g \mu_{\mathrm{B}} B_{x}$, причем значение коэффициента $g$ хорошо согласуется с известным из литературы значением $g$-фактора свободного электрона в квантовой яме такой ширины [7]. Казалось бы, это указывает на наличие в изучаемой гетероструктуре резервуара спин-поляризованных электронов. Обменное взаимодействие излучающих экситонов с поляризованными электронами должно приводить к энергетическому сдвигу экситонного пика, причем знак сдвига зависит от взаимной ориентации экситонного и электронного спинов. Во внешнем магнитном поле спины электронов прецессируют на ларморовой частоте, что приведет к осцилляциям экситонного сдвига.

Вместе с тем свободных электронов в изучаемой квантовой яме нет, как это было подтверждено в работе [2] дополнительными экспериментами. Спины электронов, связанных с дырками в неизлучающих экситонах, испытывают обменное взаимодействие с дырочными спинами, что в общем случае приводит к сильному отклонению частотной зависимости осцилляций от линейной [8]. В работе [2] сделано утверждение, что благодаря быстрой релаксации дырочных спинов в ансамбле неизлучающих экситонов спины электронов не чувствуют обменного взаимодействия с дырками на масштабах времен в десятки и сотни пикосекунд и свободно прецессируют во внешнем магнитном поле, которое на порядки меньше эффективного поля обмен-

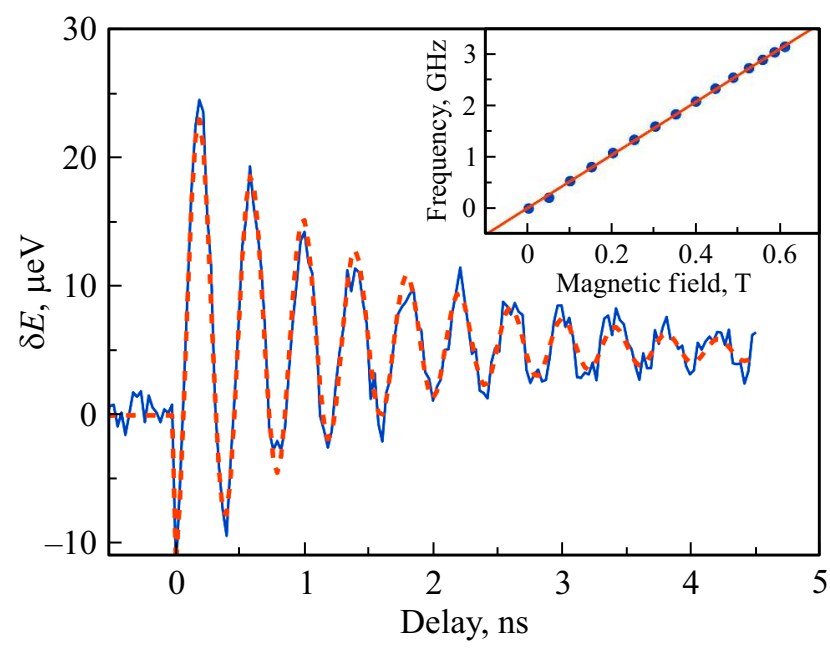

Рис. 3. Динамика разницы экситонных сдвигов, измеренных при возбуждении циркулярно поляризованными импульсами накачки в тяжелый экситон и детектировании в кои кросс-циркулярных поляризациях (сплошная зашумленная кривая). Магнитное поле $B_{x}=0.5$ Тл. Штриховая линия показывает подгонку функцией: $f(\tau)=\exp \left(-\frac{\tau}{T_{2}}\right) \cos (\omega \tau)+$ const, с параметрами: $T_{2}=1.36 \mathrm{Hc}, \omega /(2 \pi)=2.5$ ГГц. На вставке показана частота осцилляций как функция магнитного поля $B_{x}$ (точки). Сплошная линия является аппроксимацией линейной функции, $\delta E=g \mu_{\mathrm{B}} B$, где $\mu_{\mathrm{B}}-$ магнетон Бора и $g=0.365$. 
ного взаимодействия. В данной работе мы приведем подробное обоснование этого утверждения.

\section{2. Модель}

Рассмотрим обменное взаимодействие электрона и дырки в экситоне. Величина этого взаимодействия характеризуется расщеплением $\delta_{0}$ экситонных состояний с полным спином \pm 1 (светлые экситоны, если волновой вектор находится в пределах светового конуса) и полным спином \pm 2 (темные экситоны при любом волновом векторе). Оценки показывают [2], что $\delta_{0} \approx 20$ мкэВ, что много меньше, чем тепловая энергия, $k_{\mathrm{B}} T \approx 430$ мкэВ, при температуре образца $T=5 \mathrm{~K}$. Поэтому поляризация дырок в неизлучающих экситонах может релаксировать достаточно быстро [9]. Однако даже неполяризованная дырка может существенно влиять на динамику электронного спина в экситоне, поскольку обменное взаимодействие электрона с дыркой в экситоне превышает его взаимодействие с внешним магнитным полем в рассматриваемом диапазоне магнитных полей. Неполяризованные дырки создают флуктуирующее эффективное магнитное поле, которое может приводить к дефазировке прецессии электронных спинов в ансамбле. Рассмотрим этот процесс подробнее.

Спин неполяризованной дырки может равновероятно принимать значения $J= \pm 3 / 2$. Хотя среднее значение спина дырки $\langle\vec{J}\rangle=0$, ее средний квадрат отличен от нуля, $\left\langle J_{z}^{2}\right\rangle=9 / 4$. Поэтому на электрон действует флуктуирующее обменное поле дырки, которое параллельно оси $z$ и имеет среднеквадратичную амплитуду $B_{h}^{\text {eff }}$, определяемую соотношением $\delta_{0}=g_{e} \mu_{\mathrm{B}} B_{h}^{\mathrm{eff}}$, где $g_{e}-$ электронный $g$-фактор и $\mu_{\text {в }}$ - магнетон Бора. Время корреляции $\tau_{h}$, в течение которого спин электрона „чувствует“ неизменное поле $B_{h}^{\text {eff }}$, определяется двумя процессами. Во-первых, спин дырки за счет своей орбитальной компоненты эффективно связан с колебаниями решетки и изменяет свою ориентацию достаточно быстро, за время $\tau_{h 1} \sim 100$ пс [9]. Во-вторых, столкновение экситонов в резервуаре приводит к взаимному перевороту спинов носителей одного знака (либо двух электронов, либо двух дырок), входящих в экситоны. В результате этого происходит эффективное усреднение обменного поля дырок. Это хорошо известный эффект, часто называемый динамическим усреднением („,motional narrowing“ в англоязычной литературе) и наблюдаемый в различных спиновых процессах $[10,11]$. Характерное время этого процесса, $\tau_{h 2}$, в рассматриваемом нами случае можно оценить из величины уширения экситонного резонанса после импульсного возбуждения (см. рис. $2, b$ ): $\hbar \Gamma_{N R} \approx 0.1$ мэВ. Соответственно, $\tau_{h 2}=\frac{1}{\left(2 \Gamma_{N R}\right)} \approx 3$ пс. Как видно из этих оценок, эффект динамического усреднения доминирует в подавлении эффективного поля дырочных флуктуаций: $\tau_{h}=\left(1 / \tau_{h 1}+1 / \tau_{h 2}\right)^{-1} \approx \tau_{h 2} \sim 3$ пс.
Эффективное поле дырочных флуктуаций направлено вдоль оси $z$, поэтому оно не вызывает релаксации $z$-компоненты электронного спина. Релаксация компонент $S_{x}$ и $S_{y}$ в режиме динамического усреднения описывается хорошо известной формулой [11]: $1 / \tau_{x}=1 / \tau_{y}=\omega_{h}^{2} \tau_{h}$, где $\omega_{h}=\delta_{0} / \hbar=2 \pi / T_{\text {exch. }}$. Это выражение можно пояснить следующим образом. За время корреляции $\tau_{h}$ электронный спин повернется вокруг оси $z$ на угол

$$
\varphi=2 \pi \frac{\tau_{h}}{T_{\mathrm{exch}}} \ll 1,
$$

где $T_{\text {exch }}=h / \delta_{0} \approx 200$ пс. При этом $z$-компонента электронного спина, $S_{z}$, не изменится в результате такой прецессии. Компонента $S_{y}$, которая появляется в результате регулярной прецессии электронного спина во внешнем магнитном поле $B_{x}$, уменьшится за время $\tau_{h}$ на величину

$$
\delta S_{y}=S_{y} \cos \varphi-S_{y} \approx-S_{y} \frac{\varphi^{2}}{2} .
$$

Мы предполагаем, что направление вращения электронного спина в поле дырочной флуктуации на масштабах времени, больших $\tau_{h}$, никак не коррелированы между собой. Соответственно, скорость релаксации этой компоненты спина

$$
\dot{S}_{y}=\frac{\delta S_{y}}{\tau_{h}}=-\frac{1}{2} S_{y} \tau_{h}\left(\frac{2 \pi}{T_{\text {exch }}}\right)^{2}=-\frac{S_{y}}{\tau_{y}},
$$

где $\tau_{y}=\frac{2}{\tau_{h}}\left(\frac{T_{\text {exch }}}{2 \pi}\right)^{2} \approx 700$ пс - характерное время релаксации. Аналогичное выражение справедливо для $x$-компоненты спина.

Для регулярной прецессии электронного спина во внешнем магнитном поле, с учетом релаксации компоненты $S_{y}$, легко написать динамические уравнения:

$$
\begin{gathered}
\dot{S}_{z}=-\omega S_{y}, \\
\dot{S}_{y}=\omega S_{z}-\frac{S_{y}}{\tau_{y}} .
\end{gathered}
$$

Здесь частота прецессии определяется выражением $\hbar \omega=g_{e} \mu_{\mathrm{B}} B_{x}$. Исключая из этих уравнений $S_{y}$, получаем дифференциальное уравнение второго порядка для $S_{z}$ :

$$
\ddot{S}_{z}+\frac{1}{\tau_{y}} \dot{S}_{z}+\omega^{2} S_{z}=0 .
$$

Решение уравнения (5) легко находится подстановкой пробного решения в виде $S_{z}(t)=S_{z}(0) \exp (-\lambda t)$. Эта подстановка приводит к квадратичному уравнению для $\lambda$,

$$
\lambda^{2}-\frac{\lambda}{\tau_{y}}+\omega^{2}=0
$$

имеющему корни

$$
\lambda_{ \pm}=\frac{1}{2}\left[\tau_{y}^{-1} \pm \sqrt{\tau_{y}^{-2}-4 \omega^{2}}\right] .
$$


Для получения осциллируюшего решения для $S_{z}(t)$, наблюдаемого в эксперименте, необходимо, чтобы корни $\lambda_{ \pm}$содержали мнимую часть. Отсюда мы получаем условие

$$
\omega>\frac{1}{2 \tau_{y}}=\frac{1}{4} \tau_{h}\left(\frac{2 \pi}{T_{\mathrm{exch}}}\right)^{2} .
$$

Условие (8) накладывает ограничение на частоту ларморовой прецессии электронного спина во внешнем магнитном поле, при котором должно наблюдаться осциллирующее поведение спиновой поляризации:

$$
f_{L}=\frac{\omega}{2 \pi}>\frac{1}{2 \pi \cdot 2 \tau_{y}} \approx 0.1 \text { ГГц. }
$$

Это достаточно слабое ограничение по сравнению с частотами, наблюдаемыми в эксперименте (см. рис. 3). Соответствующее ограничение на внешнее магнитное поле:

$$
B_{x}=\frac{\hbar \omega}{g_{e} \mu_{\mathrm{B}}}>0.02 \text { Тл. }
$$

Отметим, что минимальное значение внешнего магнитного поля много меньше, чем эффективное поле обменного взаимодействия электрона и дырки в экситоне:

$$
B_{h}^{\mathrm{eff}}=\frac{\delta_{0}}{g_{e} \mu_{\mathrm{B}}} \approx 1 \text { Тл. }
$$

Если бы спин дырки сохранялся долгое время, то электронный спин прецессировал в суммарном поле, $B_{\text {tot }}=B_{x}+B_{h}^{\text {eff }}$, с частотой $[8,12]$ :

$$
\Omega=\sqrt{\omega^{2}+\left(\delta_{0} / \hbar\right)^{2}} \approx\left(\delta_{0} / \hbar\right)\left[1+\frac{1}{2}\left(\frac{\omega}{\delta_{0} / \hbar}\right)^{2}\right] .
$$

Следовательно, частота прецессии зависела бы нелинейным образом от внешнего магнитного поля и ограничивалась бы снизу частотой прецессии в эффективном обменном поле, $\Omega_{\text {min }}=\left(\delta_{0} / \hbar\right)=2 \pi / T_{\text {exch }} \approx 2 \pi \cdot 5$ ГГц. Наблюдаемые в эксперименте частоты лежат ниже этого предела (см. рис. 3), что говорит об эффективной деполяризации дырочного спина и эффективном усреднении его флуктуаций.

Отметим, что для случая $\omega \gg 1 /\left(2 \tau_{y}\right)$ из решения (7) получается следующая временна́я зависимость для электронной спиновой поляризации: $S_{z}(t)=S_{z}(0) \exp \left[-t /\left(2 \tau_{y}\right)\right] \cos (\omega t)$. Она хорошо описывает наблюдаемые осцилляции (см. рис. 3 ). Время затухания осцилляций $2 \tau_{y} \approx 1.4 \mathrm{нс}$, что близко к значению, полученному из подгонки кривой на рис. $3, T_{2}=1.36$ нс.

\section{3. Заключение}

Таким образом, проведенный анализ показывает, что основным процессом, приводящим к эффективному подавлению обменного взаимодействия электрона и дырки в неизлучающих экситонах, является рассеяние этих экситонов, сопровождаемое взаимным переворотом спинов носителей одного знака (либо двух электронов, либо двух дырок). Характерный масштаб времен, на которых происходит акт такого рассеяния в обсуждаемых экспериментах, составляет единицы пикосекунд. За это время электронный спин не успевает существенно изменить свое направление за счет прецессии в эффективном поле дырки. В результате рассеяния происходит эффективное усреднение дырочных спиновых флуктуаций, аналогичное эффекту динамического усреднения, обсуждаемому в литературе $[10,11]$. В результате этого спины электронов в неизлучающих экситонах прецессируют во внешнем магнитном поле практически как спины свободных электронов. Некоторые следы обменного взаимодействия сохраняются в релаксации спиновой поляризации, которая, как показывает анализ, обусловлена этим взаимодействием, а не взаимодействием с фононами.

\section{Благодарности}

Авторы благодарят Российский научный фонд за финансовую поддержку, грант № 19-72-20039, и ресурсный центр „Нанофотоника“ за предоставленный образец для исследований.

\section{Конфликт интересов}

Авторы заявляют, что у них нет конфликта интересов.

\section{Список литературы}

[1] A.V. Trifonov, S.N. Korotan, A.S. Kurdyubov, I.Ya. Gerlovin, I.V. Ignatiev, Yu.P. Efimov, S.A. Eliseev, V.V. Petrov, Yu.K. Dolgikh, V.V. Ovsyankin, A.V. Kavokin. Phys. Rev. B, 91, 115307 (2015).

[2] A.V. Trifonov, E.S. Khramtsov, K.V. Kavokin, I.V. Ignatiev, A.V. Kavokin, Y.P. Efimov, S.A. Eliseev, P.Yu. Shapochkin, M. Bayer. Nanosecond Spin Coherence Time of Nonradiative Excitons in GaAs/AlGaAs Quantum Wells. Phys. Rev. Lett., 122, 147401 (2019).

[3] E.L. Ivchenko. Optical Spectroscopy of Semiconductor Nanostructures (Springer, Berlin, 2004).

[4] E.S. Khramtsov, P.A. Belov, P.S. Grigoryev, I.V. Ignatiev, S.Yu. Verbin, Yu.P. Efimov, S.A. Eliseev, V.A. Lovtcius, V.V. Petrov, S.L. Yakovlev. J. Appl. Phys., 119, 184301 (2016).

[5] P.S. Grigoryev, A.S. Kurdyubov, M.S. Kuznetsova, I.V. Ignatiev, Yu.P. Efimov, S.A. Eliseev, V.V. Petrov, V.A. Lovt'cius, P.Yu. Shapochkin. Superlat. Microstr., 97, 452 (2016).

[6] E.S. Khramtsov, P.S. Grigoryev, D.K. Loginov, I.V. Ignatiev, Yu.P. Efimov, S.A. Eliseev, P.Yu. Shapochkin, E.L. Ivchenko, M. Bayer. Phys. Rev. B, 99, 035431 (2019).

[7] I.A. Yugova, A. Greilich, D.R. Yakovlev, A.A. Kiselev, M. Bayer, V.V. Petrov, Yu.K. Dolgikh, D. Reuter, A.D. Wieck. Phys. Rev. B, 75, 245302 (2007).

[8] M. Dyakonov, X. Marie, T. Amand, P.Le Jeune, D. Robart, M. Brousseau, J. Barrau. Phys. Rev. B, 56, 10412 (1997). 
[9] R.T. Harley, A. Balocchi, T. Amand. Spin Dynamics of Free Carriers in Quantum Wells. In: Spin Physics in Semiconductors, ed. by M.I. Dyakonov (Springer 2017, 2nd edn, Chap. 2, p. 59).

[10] А. Абрагам. Ядерный магнетизм (М., Изд-во. Иностр. лит., 1963). [А. Abragam. The Principle of Nuclear Magnetism, Oxford Clarendon Press, 1961].

[11] M.I. Dyakonov. Basic of Semiconductors and Spin Physics. In: Spin Physics in Semiconductors, ed. by M.I. Dyakonov (Springer 2017, 2nd edn, Chap. 1, p. 21).

[12] I.Ya. Gerlovin, Yu.K. Dolgikh, S.A. Eliseev, V.V. Ovsyankin, Yu.P. Efimov, V.V. Petrov, I.V. Ignatiev, I.E. Kozin, Y. Masumoto. Phys. Rev. B, 65, 035317 (2001).

Редактор Г.А. Оганесян

\section{Suppression of electron-hole exchange interaction in reservoir of nonradiative excitons}

A.V. Trifonov, I.V. Ignatiev, K.V. Kavokin, A.V. Kavokin, P.Yu. Shapochkin, Yu.P. Efimov, S.A. Eliseev,

V.A. Lovtcius

St. Petersburg State University, 198504 St. Petersburg, Russia

Abstract Mechanisms of suppression of the electron-hole exchange interaction in nonradiative excitons with large in-plane wave vector in high-quality heterostructures with quantum wells are analyzed theoretically. It is shown that the dominating suppression mechanism is the exciton-exciton scattering accompanied by the mutual spin flips of like carriers (either two electrons or two holes), comprising the excitons. As a result, the electron spin polarization in nonradiative excitons may survive for a long time. The analysis of experimental data shows that this relaxation time can exceed one nanosecond. This long and optically controllable spin memory in the exciton reservoir may be of interest for future information technologies. 